\title{
Analysis of the Time-To-Onset and Factors Affecting Clinical Outcomes of Immune Reconstitution Inflammatory Syndrome in People Living with HIV Using Data from the Japanese Spontaneous Reporting Database
}

\author{
Hiroyuki Tanaka ${ }^{1}$, Tatsuhiko Wada ${ }^{2}$, Katsuhiro Ohyama ${ }^{3}$, and Toshihiro Ishii ${ }^{1}$ \\ ${ }^{1}$ Department of Practical Pharmacy, Faculty of Pharmaceutical Sciences, Toho University, Miyama, Funabashi, Chiba, \\ Japan; ${ }^{2}$ Department of Rheumatology and Infectious Diseases, Kitasato University School of Medicine, Kitasato, \\ Minami-ku, Sagamihara, Kanagawa, Japan; ${ }^{3}$ Center for Experiential Pharmacy Practice, School of Pharmacy, Tokyo \\ University of Pharmacy and Life Sciences, Horinouchi, Hachioji, Tokyo, Japan.
}

Corresponding Author: Hiroyuki Tanaka, Department of Practical Pharmacy, Faculty of Pharmaceutical Sciences, Toho University, 2-2-1 Miyama, Funabashi, Chiba 274-8510, Japan; email: hiroyuki.tanaka@phar.toho-u.ac.jp

Received, December 21, 2020; Revised, March 29, 2021; Accepted, March 31, 2021; Published, April 3, 2021

\begin{abstract}
Purpose: Data on immune reconstitution inflammatory syndrome (IRIS), despite being a widely recognized complication of antiretroviral therapy, remain limited. The objective of the present study was to evaluate the time-to-onset and factors affecting clinical outcomes of IRIS in people living with HIV (PLWH) using data from the Japanese Adverse Drug Event Report (JADER) database. Methods: Data of PLWH who developed IRIS as an adverse event were extracted from the JADER database. Cases with the data of both the start date of anti-HIV drug therapy and date of IRIS onset were included in the study. The survey items included sex, age, anti-HIV drug use, IRIS-compatible events, time-to-onset of IRIS, and clinical outcome. The time-to-onset of IRIS was evaluated in relation to anchor drug use. Overall, 79 cases were included in the analysis. Results: The median (range) time-to-onset of IRIS was 29 (1-365) days, and it differed significantly between IRIS-compatible events $(P=0.029)$. In particular, the time-to-onset of Pneumocystis pneumonia-IRIS was the shortest among the IRIS-compatible events (median [range]: 12 [5301] days). Age $\geq 50$ years at IRIS onset appeared to be related to the poor clinical outcomes of IRIS in PLWH $(P=0.048)$. The use of integrase strand transfer inhibitors did not affect the time-to-onset of IRIS or clinical outcome of IRIS in PLWH. Conclusion: This analysis based on the data from the JADER database revealed that IRIS-compatible events were related to the time-to-onset of IRIS and that patients older than 50 years had poorer clinical outcomes of IRIS. This finding will be useful for healthcare professionals when considering medications for patients with HIV infection/AIDS and for the management of IRIS.
\end{abstract}

\section{INTRODUCTION}

Antiretroviral therapy (ART) reduces plasma HIVviral load (VL) and improves the CD4+ T-cell count in people living with HIV (PLWH) $(1,2)$. The immunological changes induced by ART are associated with a reduced frequency of opportunistic infections (OIs) and prolonged survival. Consequently, in recent years, the life expectancy of PLWH has gradually improved to that of populations without HIV (3). However, ART can elicit an inflammatory response, referred to as immune reconstitution inflammatory syndrome (IRIS) (4). IRIS results from an exaggerated inflammatory reaction to various antigenic stimuli following the initiation of ART (4). IRIS can manifest as "paradoxical" IRIS, in which an OI diagnosed before ART initially responds to treatment but worsens after ART initiation, or "unmasking" IRIS, in which a disease that is not clinically evident before ART is induced upon its initiation, often with unusual or florid inflammatory features in association with rapid restoration of pathogen-specific immune responses (5). Although IRIS is a well-established entity, there are uncertainties regarding its etiology and management. Several studies have reported the incidence of IRIS and identified risk factors for its onset, including low CD4+ T-cell count, high plasma HIV-VL, the magnitude and rate of CD4+ T-cell reconstitution, and plasma HIV-VL reduction following ART initiation (5-10). However, only a few studies have focused on the time-to-onset and factors affecting clinical outcome of IRIS, particularly, in Japan.

In recent years, studies have utilized data from the Japan Adverse Drug Event Reporting (JADER) database $(11,12)$, a national spontaneous reporting database in Japan. As of December 2019, the JADER database contained reports of more than 500,000 specific patients, including many involving PLWH. 
In addition, many records in this database include the start date of drug administration and/or the date of adverse event occurrence.

In the present study, we searched the JADER database and analyzed the data to clarify the time-toonset and factors affecting clinical outcomes of IRIS in PLWH.

\section{METHODS}

\section{Data source and survey content}

Data available in the JADER database between April 2004 and October 2018 were obtained from the Pharmaceuticals and Medical Devices Agency (PMDA)

(https://www.pmda.go.jp/safety/infoservices/drugs/adr-info/suspected-adr/0003.html).

The JADER database has four data sets: patient demographic information (demo), drug information (drug), adverse event (reac), and medical history (hist). In the JADER database, age is rounded to the nearest 10 units. For this analysis, we defined "young" individuals as those assigned to the "20s," "30s," and "40s," and "older" individuals as those assigned to the "50s," "60s," "70s," "80s," "90s," and "100s" based on the WHO/CDC definition for older age (13). This is because there is some evidence to suggest that HIV infection accelerates at least some of the agerelated processes (14-16). Missing data were denoted as "unknown." In some cases, age was registered as being within a particular age group (for example, newborn, infant, or older) or a period of pregnancy, and data for such cases were also denoted as "unknown." The adverse events in "reac" are based on preferred terms (PT) in the Medical Dictionary for Regulatory Activities/Japanese version (MedDRA/J).

Data in which PLWH who developed IRIS as an adverse event were extracted from the overall data. Among these data, cases that included both the start date of anti-HIV drug therapy and the date of IRIS onset were surveyed. The following adverse events related to IRIS in MedDRA/J version 21.0 were adopted: "Mycobacterium avium complex immune restoration disease" (PT code: 10058449), "Immune reconstitution inflammatory syndrome" (PT code: 10065042), "Immune reconstitution inflammatory syndrome-associated Kaposi's sarcoma" (PT code: 10072796), and "Immune reconstitution inflammatory syndrome-associated tuberculosis" (PT code: 10072797). The survey parameters included sex, age, anti-HIV drug use, IRIScompatible event, time-to-onset of IRIS, and clinical outcome.

In cases where IRIS was reported as an adverse event, other adverse events reported on the same day were regarded as IRIS-compatible events, and when no adverse event was reported on the same day, it was regarded as "unknown." Conversely, in cases where "Mycobacterium avium complex immune restoration disease," "Immune reconstitution inflammatory syndrome-associated Kaposi's sarcoma," or "Immune reconstitution inflammatory syndrome-associated tuberculosis" were reported as an adverse event, nontuberculous mycobacteria (NTM) infection, Kaposi's sarcoma (KS), and tuberculosis (TB) were considered IRIS-compatible events, respectively.

The time-to-onset of IRIS was evaluated in relation to the use of anchor drug and was calculated as follows: "date of IRIS onset" - "date of start of anchor drug" +1 (Figure 1). A maximum of 365 days was used as the date of onset when the period to IRIS onset was 1 year or more. Protease inhibitors (PIs) (atazanavir, darunavir, fosamprenavir, indinavir, lopinavir, and nelfinavir), non-nucleoside reverse transcriptase inhibitors (NNRTIs) (efavirenz, nevirapine, and rilpivirine), and integrase strand transfer inhibitors (INSTIs) (dolutegravir, elvitegravir, and raltegravir) were adopted as anchor drugs.

Any patient who developed two IRIScompatible events on different days was counted as two cases. For reported clinical outcomes of IRIScompatible events, we considered "unrecovered," "death," or "sequelae," to represent cases with "poor outcomes," and "recovery" or "remission" to represent cases with "good outcomes."

\section{Statistical analysis}

Time-to-onset of IRIS among groups (sex, age group, anchor drug class, and IRIS-compatible events) was compared using the Wilcoxon rank-sum test or Kruskal-Wallis test. For analysis of factors affecting clinical outcomes in patients with IRIS, Fisher's exact test was used. For factors with $P$-value $<0.1$, multivariate logistic regression analyses were subsequently conducted to determine odds ratios for poor outcomes. EZR (Saitama Medical Center, Jichi Medical University, Saitama, Japan) (17), a graphical user interface for R (The R Foundation for Statistical Computing, Vienna, Austria) was used. It is a modified version of $\mathrm{R}$ commander designed to add statistical functions $(P<0.05)$.

\section{RESULTS}

\section{Study population}

Between April 2004 and October 2018, 305 IRIS cases involving PLWH were reported, of which 79 met the criteria of this study (Figure 2). The 

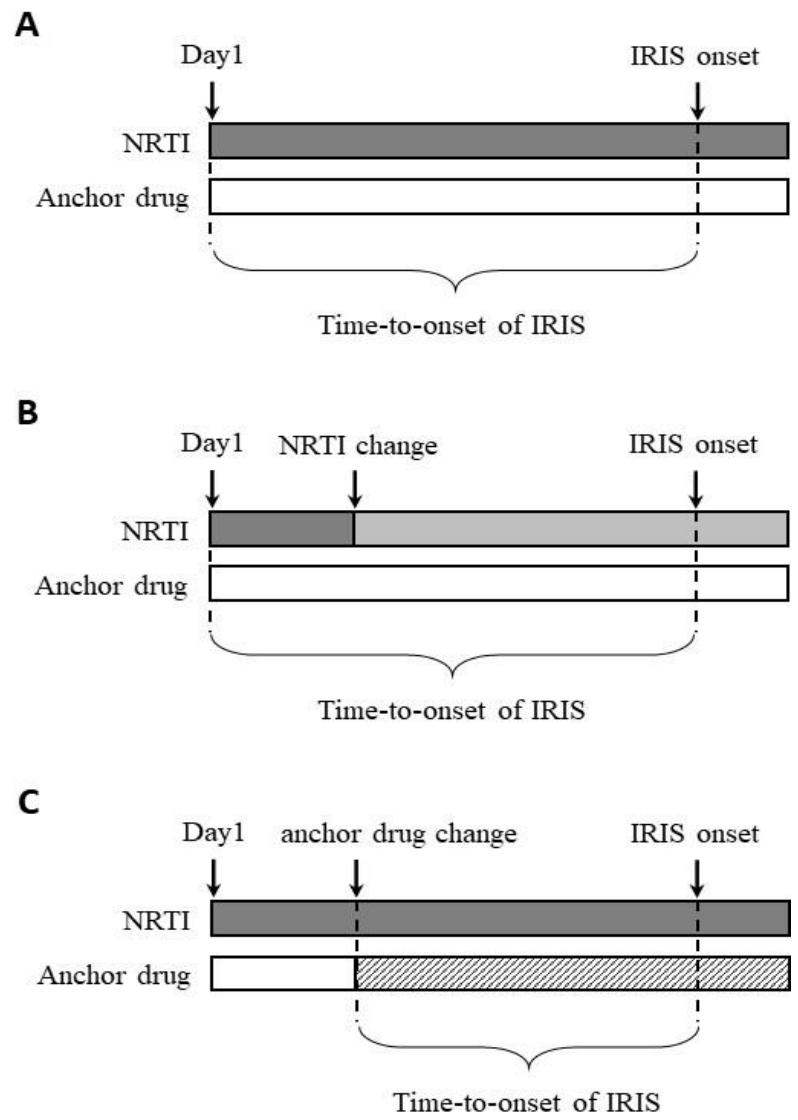

Figure 1. Method used to calculate the time-to-onset of IRIS. A, NRTI and the anchor drug were initiated or changed at the same time; B, NRTI was changed; C, the anchor drug was changed. Keys: IRIS, immune reconstitution inflammatory syndrome; NRTI, nucleoside/nucleotide reverse transcriptase inhibitor.

characteristics of these cases are shown in Table 1. Males accounted for 73 cases $(92.4 \%)$ and older patients accounted for $55(69.6 \%)$ cases. PI (39 cases [49.4\%]) was the most frequently used anchor drug at IRIS onset. However, in recent years, INSTIs accounted for a majority of IRIS cases (Figure 3). The most frequently reported IRIS-compatible events were NTM infection (13 cases) followed by cytomegalovirus (CMV) infection (12 cases) and Pneumocystis pneumonia (PCP) (11 cases). There were three cases of central nervous system (CNS)IRIS (cerebral toxoplasmosis, 2 cases; central nervous system tuberculosis, 1 case). The IRIScompatible event was not reported in 16 cases $(20.3 \%)$. Ten cases $(12.7 \%)$ had a poor clinical outcome.

\section{Comparison of the time-to-onset of IRIS}

In all cases $(\mathrm{n}=79)$, the median (range) time-toonset of IRIS was 29 (1-365) days. Table 2 shows the time-to-onset of IRIS by sex, age group, anchor drug class, and IRIS-compatible event. A comparison of the time-to-onset of IRIS by sex and age revealed no significant differences. There was no significant difference in the time-to-onset of IRIS among the various anchor drugs used. However, the use of PI tended to increase the time-to-onset of IRIS compared with the use of other anchor drugs. The time-to-onset of IRIS differed significantly between IRIS-compatible events $(P=0.029)$. Particularly, the median (range) time-to-onset of PCP was 12 (5-301) days, which was the shortest among all IRIScompatible events: NTM infection (median [range] 13 [1-250] days), CMV infection (34.5 [2-80] days), hepatitis B (HB) (50 [29-302] days), herpes zoster (HZ) (78 [37-134] days), TB (14 [14-238] days), and KS (74 [14-143] days).

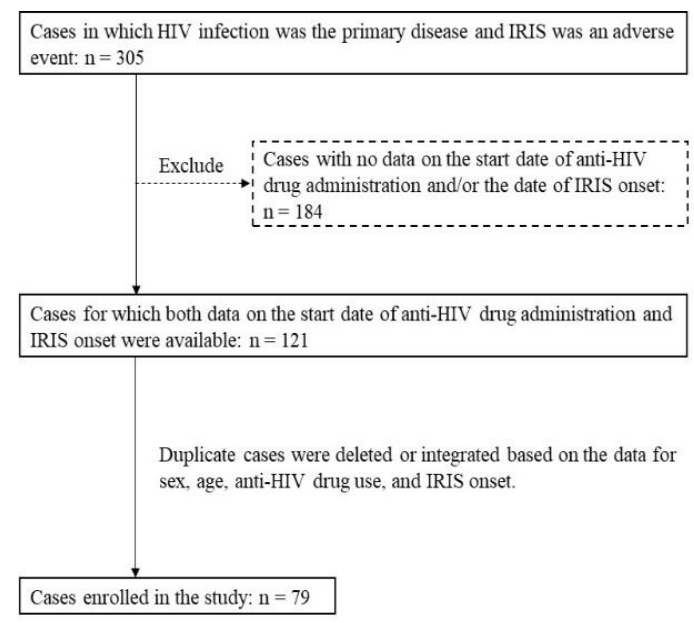

Figure 2. Procedure for selecting cases for analysis. IRIS: immune reconstitution inflammatory syndrome

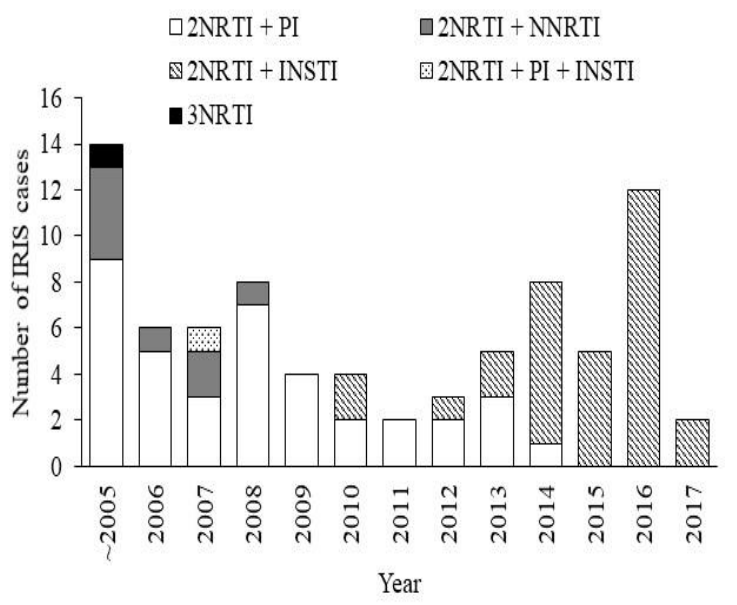

Figure 3. Temporal trends in the onset of IRIS and the ART regimens used. IRIS, immune reconstitution inflammatory syndrome; NRTI, nucleoside/nucleotide reverse transcriptase inhibitor; PI, protease inhibitor; NNRTI, nonnucleoside reverse transcriptase inhibitor; INSTI, integrase strand transfer inhibitor. 
Table 1. Characteristics of the 79 IRIS cases identified in the JADER database.

\begin{tabular}{|c|c|}
\hline Characteristic & Cases, n (\%) \\
\hline \multicolumn{2}{|l|}{ Sex } \\
\hline Male & $73(92.4)$ \\
\hline Female & $6(7.6)$ \\
\hline \multicolumn{2}{|l|}{ Age group $^{a}$} \\
\hline Young & $55(69.6)$ \\
\hline Older & $16(20.3)$ \\
\hline \multicolumn{2}{|l|}{ ART regimen } \\
\hline 2NRTI + PI & $38(48.1)$ \\
\hline 2NRTI + NNRTI & $8(10.1)$ \\
\hline 2NRTI + INSTI & $31(39.2)$ \\
\hline 2NRTI + PI + INSTI & $1(1.3)$ \\
\hline 3NRTI & $1(1.3)$ \\
\hline \multicolumn{2}{|l|}{ IRIS-compatible event ${ }^{b}$} \\
\hline NTM infection & $13(16.5)$ \\
\hline CMV infection & $12(15.2)$ \\
\hline $\mathrm{PCP}$ & $11(13.9)$ \\
\hline $\mathrm{HB}$ & $7(8.9)$ \\
\hline $\mathrm{HZ}$ & $4(5.1)$ \\
\hline TB & $3(3.8)$ \\
\hline KS & $3(3.8)$ \\
\hline Others/Unknown & $27(34.2)$ \\
\hline \multicolumn{2}{|c|}{ Clinical outcomes of IRIS } \\
\hline Recovery & $26(32.9)$ \\
\hline Remission & $43(54.4)$ \\
\hline Sequelae & $0(0.0)$ \\
\hline Unrecovered & $9(11.4)$ \\
\hline Death & $1(1.3)$ \\
\hline \multicolumn{2}{|c|}{$\begin{array}{l}\text { "ata for age were missing for eight cases. }{ }^{\mathrm{b}} \text { In one case } \\
\text { HB and KS developed as IRIS on the same day. Therefore } \\
\text { the sum of the percentages is not } 100 \text {. ART, antiretrovira } \\
\text { therapy; NRTI, nucleoside/nucleotide reverse } \\
\text { transcriptase inhibitor; PI, protease inhibitor; NNRTI } \\
\text { non-nucleoside reverse transcriptase inhibitor; INSTI } \\
\text { integrase strand transfer inhibitor; NTM, nontuberculous } \\
\text { mycobacteria; CMV, cytomegalovirus; PCP } \\
\text { Pneumocystis pneumonia; HB, hepatitis B; HZ, herpes } \\
\text { zoster; TB, tuberculosis; KS, Kaposi's sarcoma }\end{array}$} \\
\hline
\end{tabular}

\section{Factors affecting the clinical outcome of IRIS}

Table 3 shows the results of the univariate and multivariate analyses of factors affecting the clinical outcomes of IRIS. The univariate analysis revealed that older patients (50 years and over) and the use of PI were factors related to poor outcomes $(P=0.040$, $P=0.048$, respectively). There was no significant difference in the time-to-onset of IRIS between the poor outcome and good outcome groups. However, the poor outcome group tended to include a higher proportion of patients with a time-to-onset of IRIS of 14 days or less. A multivariate logistic regression analysis using a forced entry method was performed to examine factors with $P$-values $<0.1$ in the univariate analysis. A significant difference was found in the older individuals (50 years and older) (adjusted odds ratio: 4.353, 95\% confidence interval [CI]: 1.014-19.116, $P=0.048$ ) (Table 2). The use of PI was a significant factor in the univariate analysis but not in the multivariate analysis.

\section{DISCUSSION}

This analysis based on data from the JADER database revealed that IRIS-compatible events were related to the time-to-onset of IRIS and that older patients had poor clinical outcomes of IRIS. A metaanalysis of cohort studies concluded that IRIS occurs in $13 \%$ of PLWH following the effective initiation of ART (18). In addition, it has been reported that IRIS onset may be associated with death or hospitalization $(19,20)$. Therefore, identification of the time-toonset and factors affecting clinical outcomes of IRIS is of high clinical relevance. To clarify these factors, we searched the JADER database and analyzed the extracted data.

We found that IRIS-compatible events were related to the time-to-onset of IRIS. Particularly, the time-to-onset of PCP-IRIS was the shortest relative to other IRIS-compatible events (median [range] 12 [5-301] days). The time-to-onset of PCP-IRIS was similar to that reported previously (21). These results alert healthcare professionals about the rapid development of PCP-IRIS following the initiation of ART. In addition, the present study revealed the time-to-onset of several other IRIS-compatible events. Information about the timing of each IRIScompatible event will significantly contribute to its appropriate treatment.

In the present study, age $\geq 50$ years at IRIS onset was found to be a factor related to poor clinical outcomes. Ratnam et al. reported that patients with an IRIS event, compared with patients without an IRIS event, were younger at the time of ART initiation (8). Thus, the factors affecting the onset and clinical outcome of IRIS may differ. Several studies have shown that older patients with HIV who initiate ART are less responsive to CD4+ T cells compared with younger patients (22-24). The difference in the speed and extent of CD4+ T-cell recovery with age may affect the onset and clinical prognosis of IRIS. Therefore, careful observation and strict management are needed for older patients at IRIS onset. Conversely, an IRIS-compatible event was not identified as a factor associated with the clinical outcomes of IRIS in the present study. This 
Table 2. Comparison of the time-to-onset of IRIS among different patient groups.

\begin{tabular}{lccc}
\hline Characteristic & Number of cases & Median days (range) & $\boldsymbol{P}$-value \\
\hline Sex & 73 & & 0.667 \\
$\quad$ Male & 6 & $29(1-365)$ & \\
$\quad$ Female & & $31.5(8-250)$ & 0.773 \\
Age group & & \\
$\quad$ Young & 55 & $32(1-365)$ & \\
$\quad$ Older & 16 & $39.5(7-302)$ & 0.071 \\
Anchor drug class & & & \\
PI & & $40(1-302)$ & \\
NNRTI & 39 & $11.5(2-238)$ & \\
INSTI & 8 & $20(5-365)$ & \\
IRIS-compatible event & 32 & & \\
NTM infection & & $13(1-250)$ & \\
CMV infection & 13 & $34.5(2-80)$ & \\
PCP & 12 & $12(5-301)$ & \\
HB & 11 & $50(29-302)$ & \\
HZ & 7 & $78(37-134)$ & \\
TB & 4 & $14(14-238)$ & \\
KS & 3 & $74(14-143)$ & \\
\hline
\end{tabular}

${ }^{\mathrm{a}}$ Data for age were missing for eight cases. ${ }^{\mathrm{b}}$ In one case, PI + INSTI was used as the anchor drug, and in one other case the 3NRTI regimen was used. IRIS, immune reconstitution inflammatory syndrome. PI, protease inhibitor; NNRTI, non-nucleoside reverse transcriptase inhibitor; INSTI, integrase strand transfer inhibitor; NTM, nontuberculous mycobacteria; CMV, cytomegalovirus; PCP, Pneumocystis pneumonia; HB, hepatitis B; HZ, herpes zoster; TB, tuberculosis; KS, Kaposi's sarcoma. ${ }^{*} P<0.05$ (Kruskal-Wallis test)

may have been due to the research methods and/or regions employed. Typically, CNS-IRIS has been reported to associated with a high fatality rate (25). The 79 cases analyzed included a small number of CNS-IRIS cases (cerebral toxoplasmosis, 2 cases; central nervous system tuberculosis, 1 case), and no cases of progressive multifocal leukoencephalopathy or cryptococcal meningitis. These results may have influenced the analysis of factors affecting the clinical outcomes of IRIS.

A relationship between INSTI use and IRIS onset has been suspected (26-28). INSTIs may lead to a rapid reduction in HIV-VL. Our study revealed that INSTIs were used more frequently in patients who had recently developed IRIS (after 2014). However, these results may be related to the increased use of INSTIs in Japan (29). The INSTI use group tended to have a shorter time to IRIS onset than the PI use group, although the difference between groups was not significant. This may be partially related to the rapid viral suppression by INSTIs, and the use of INSTIs did not affect the clinical outcome of IRIS. Conversely, the univariate analysis revealed that the PI use was associated with poor clinical outcomes. INSTIs have become the cornerstone of PLWH treatment. PIs, which lead to a slow CD4+ T-cell recovery and gradual HIV-VL decline compared with INSTI, may be selected for PLWH at a high risk of IRIS development. However, the multivariate analysis revealed that the use of PI was not a risk factor for poor clinical outcomes. Our study covered a long period, between April 2004 and October 2018, which represents the time when the treatment guidelines for HIV and the prevention/treatment guidelines for OIs differed from those available today. Therefore, our results may have been influenced by these differences.

Spontaneous reporting systems such as the JADER database have limitations, including a lack of details for assessing causal relationships, a generalized underreporting bias, the relationship between reporting rate and the period of drug availability, and the quality of the data (i.e., missing data, "extreme duplication," and multiple records) (30). The JADER database does not include laboratory data such as the CD4+ T-cell count or plasma HIV-VL. It was also difficult to determine the treatment status of OIs before ART initiation, which was necessary to determine "paradoxical" IRIS and "unmasking" IRIS. Therefore, we were unable to determine whether these factors affect the time-toonset and clinical outcome of IRIS. As some risk factors have been shown to affect the incidence of IRIS or the associated mortality and may be related to the time-to-onset and clinical outcome of IRIS, the results should be interpreted considering these limitations. However, despite the unique limitations of spontaneous reporting systems for adverse events, 
our study revealed the time-to-onset and factors affecting clinical outcomes of IRIS in PLWH.

\section{CONCLUSIONS}

Despite limitations inherent to the database, our analysis of the JADER database indicated that IRIScompatible events appeared to be a factor affecting the time-to-onset of IRIS and that age at IRIS onset appeared to be a factor affecting the clinical outcome.
We hope that this information will be useful for healthcare professionals when considering medication for patients with HIV infection/AIDS and for the management of IRIS. Further extensive studies are needed to validate these results.

Table 3. Analysis of factors affecting clinical outcomes of IRIS.

\begin{tabular}{|c|c|c|c|c|c|c|}
\hline \multirow[t]{2}{*}{ Factor } & \multicolumn{3}{|c|}{ Univariate analysis } & \multicolumn{3}{|c|}{ Multivariate analysis } \\
\hline & $\begin{array}{c}\text { Good } \\
\text { outcome } \\
n=69\end{array}$ & $\begin{array}{c}\text { Poor } \\
\text { outcome } \\
n=10\end{array}$ & $p$ & $\begin{array}{l}\text { Adjusted } \\
\text { odds ratio }\end{array}$ & $95 \% \mathrm{CI}$ & $p$ \\
\hline \multicolumn{7}{|l|}{ Sex } \\
\hline Male & 63 & 10 & 1.000 & & & \\
\hline \multicolumn{7}{|l|}{ Age group ${ }^{a}$} \\
\hline Older & 11 & 5 & $0.040^{*}$ & 4.353 & $1.014-19.116$ & $0.048^{+}$ \\
\hline \multicolumn{7}{|c|}{ Anchor drug class ${ }^{b}$} \\
\hline PI & 31 & 8 & $0.048^{*}$ & 3.691 & $0.802-26.563$ & 0.097 \\
\hline NNRTI & 8 & 0 & 0.586 & & & \\
\hline INSTI & 30 & 2 & 0.189 & & & \\
\hline \multicolumn{7}{|c|}{ Time-to-onset of IRIS } \\
\hline Within 14 days & 21 & 5 & 0.284 & & & \\
\hline \multicolumn{7}{|c|}{ IRIS-compatible event } \\
\hline NTM infection & 10 & 3 & 0.355 & & & \\
\hline CMV infection & 11 & 1 & 1.000 & & & \\
\hline PCP & 11 & 0 & 0.342 & & & \\
\hline HB & 7 & 0 & 0.587 & & & \\
\hline $\mathrm{HZ}$ & 4 & 0 & 1.000 & & & \\
\hline TB & 3 & 0 & 1.000 & & & \\
\hline $\mathrm{KS}$ & 3 & 0 & 1.000 & & & \\
\hline
\end{tabular}

${ }^{\mathrm{a}}$ Data for age were missing for eight cases. ${ }^{\mathrm{b}}$ In one case, PI + INSTI was used as the anchor drug, and in one case the 3NRTI regimen was used. IRIS, immune reconstitution inflammatory syndrome; PI, protease inhibitor; NNRTI, nonnucleoside reverse transcriptase inhibitor; INSTI, integrase strand transfer inhibitor; NTM, nontuberculous mycobacteria; CMV, cytomegalovirus; PCP, Pneumocystis pneumonia; HB, hepatitis B. ${ }^{*} p<0.05$ (Fisher's exact test); ${ }^{\dagger} p<0.05$ (multivariate logistic regression analyses).

FUNDING. This research received no specific grant from any funding agency in the public, commercial, or not-for-profit sectors.

CONFLICT OF INTEREST. The authors have no conflicts of interest to declare.

PROTOCOL ETHICS APPROVAL AND PATIENT CONSENT. Not applicable.

\section{REFERENCES}

1. Palella FJ Jr, Delaney KM, Moorman AC, Loveless MO, Fuhrer J, Satten GA, Aschman DJ, Holmberg SD. Declining morbidity and mortality among patients with advanced human immunodeficiency virus infection. HIV
Outpatient Study Investigators. N Engl J Med, 1998; 338: 853-860. DOI: 10.1056/NEJM199803263381301.

2. Lederman MM. Immune restoration and CD4+ T-cell function with antiretroviral therapies. AIDS, 2001; 15: S11-S15. DOI: 10.1097/00002030-200102002-00003.

3. Antiretroviral Therapy Cohort Collaboration. Survival of HIV-positive patients starting antiretroviral therapy between 1996 and 2013: a collaborative analysis of cohort studies. Lancet HIV, 2017; 4: e349-e356. DOI: 10.1016/S23523018(17)30066-8.

4. French MA, Lenzo N, John M, Mallal SA, McKinnon EJ, James IR, Price P, Flexman JP, Tay-Kearney ML. Immune restoration disease 
after the treatment of immunodeficient HIVinfected patients with highly active antiretroviral therapy. HIV Med, 2000; 1: 107115. DOI: 10.1046/j.1468-1293.2000.00012.x.

5. Haddow LJ, Moosa MY, Mosam A, Moodley P, Parboosing R, Easterbrook PJ. Incidence, clinical spectrum, risk factors and impact of HIV-associated immune reconstitution inflammatory syndrome in South Africa. PLoS One, 2012; 7: e40623. DOI: 10.1371/journal.pone.0040623.

6. Shelburne SA, Visnegarwala F, Darcourt J, Graviss EA, Giordano TP, White AC Jr, Hamill RJ. Incidence and risk factors for immune reconstitution inflammatory syndrome during highly active antiretroviral therapy. AIDS, 2005; 19: 399-406. DOI: 10.1097/01.aids.0000161769.06158.8a.

7. Jevtović DJ, Salemović D, Ranin J, Pesić I, Zerjav S, Djurković-Djaković O. The prevalence and risk of immune restoration disease in HIV-infected patients treated with highly active antiretroviral therapy. HIV Med, 2005; 6: 140-143. DOI: $10.1111 / \mathrm{j} .1468-$ 1293.2005.00277.x.

8. Ratnam I, Chiu C, Kandala NB, Easterbrook PJ. Incidence and risk factors for immune reconstitution inflammatory syndrome in an ethnically diverse HIV type 1-infected cohort. Clin Infect Dis, 2006; 42: 418-427. DOI: 10.1086/499356.

9. Murdoch DM, Venter WD, Feldman C, Van Rie A. Incidence and risk factors for the immune reconstitution inflammatory syndrome in HIV patients in South Africa: a prospective study. AIDS, 2008; 22: 601-610. DOI: 10.1097/QAD.0b013e3282f4a607.

10. Grant PM, Komarow L, Andersen J, Sereti I, Pahwa S, Lederman MM, Eron J, Sanne I, Powderly W, Hogg E, Suckow C, Zolopa A. Risk factor analyses for immune reconstitution inflammatory syndrome in a randomized study of early vs. deferred ART during an opportunistic infection. PLoS One, 2010; 5: e11416. DOI: 10.1371/journal.pone.0011416.

11. Komada F, Nakayama Y, Takara K. Analysis of time-to-onset and onset-pattern of interstitial lung disease after the administration of monoclonal antibody agents. Yakugaku Zasshi, 2018; 138: 1587-1594. DOI: 10.1248/yakushi.18-00094.

12. Tanaka H, Yoshiba Y, Watanabe T, Satoh M, Ishii T. Analysis of patients with hypomagnesemia using the Japanese Adverse Drug Event Report database (JADER). J Pharm
Pharm Sci, 2018; 21: 46-53. DOI: 10.18433/jpps29692.

13. Centers for Disease Control and Prevention. AIDS among persons aged greater than or equal to 50 years -- United States, 1991-1996. MMWR, 1998; 47: 21-27.

14. Appay V, Almeida JR, Sauce D, Autran B, Papagno L. Accelerated immune senescence and HIV-1 infection. Exp Gerontol, 2007; 42: 432-437. DOI: 10.1016/j.exger.2006.12.003.

15. Effros RB, Fletcher CV, Gebo K, Halter JB, Hazzard WR, Horne FM, et al. Aging and infectious diseases: workshop on HIV infection and aging: what is known and future research directions. Clin Infect Dis, 2008; 47: 542-553. DOI: 10.1086 / 590150

16. Rickabaugh TM, Baxter RM, Sehl M, Sinsheimer JS, Hultin PM, Hultin LE, Quach A, Martínez-Maza O, Horvath S, Vilain E, Jamieson BD. Acceleration of age-associated methylation patterns in HIV-1-infected adults. PLoS One, 2015; 10: e0119201. DOI: 10.1371/journal.pone.0119201.

17. Kanda Y. Investigation of the freely available easy-to-use software "EZR" for medical statistics. Bone Marrow Transplant, 2013; 48: 452-458. DOI: 10.1038/bmt.2012.244.

18. Müller M, Wandel S, Colebunders R, Attia S, Furrer H, Egger M; IeDEA Southern and Central Africa. Immune reconstitution inflammatory syndrome in patients starting antiretroviral therapy for HIV infection: a systematic review and meta-analysis. Lancet Infect Dis, 2010; 10: 251-261. DOI: 10.1016/S1473-3099(10)70026-8.

19. Hoyo-Ulloa I, Belaunzarán-Zamudio PF, Crabtree-Ramirez B, Galindo-Fraga A, PérezAguinaga ME, Sierra-Madero JG. Impact of the immune reconstitution inflammatory syndrome (IRIS) on mortality and morbidity in HIVinfected patients in Mexico. Int J Infect Dis, 2011; 15: e408-414. DOI: 10.1016/j.ijid.2011.02.007.

20. Novak RM, Richardson JT, Buchacz K, Chmiel JS, Durham MD, Palella FJ, Wendrow A, Wood K, Young B, Brooks JT; HIV Outpatient Study (HOPS) Investigators. Immune reconstitution inflammatory syndrome: incidence and implications for mortality. AIDS, 2012; 26: 721-730. DOI: 10.1097/QAD.0b013e3283511e91.

21. Mok HP, Hart E, Venkatesan P. Early development of immune reconstitution inflammatory syndrome related to Pneumocystis pneumonia after antiretroviral 
therapy. Int J STD AIDS, 2014; 25: 373-377. DOI: $10.1177 / 0956462413506888$.

22. Manfredi R, Chiodo F. A case-control study of virological and immunological effects of highly active antiretroviral therapy in HIV-infected patients with advanced age. AIDS, 2000; 14: 1475-1477. DOI: 10.1097/00002030200007070-00034.

23. Viard JP, Mocroft A, Chiesi A, Kirk O, Røge B, Panos G, Vetter N, Bruun JN, Johnson M, Lundgren JD; EuroSIDA Study Group. Influence of age on CD4 cell recovery in human immunodeficiency virus-infected patients receiving highly active antiretroviral therapy: evidence from the EuroSIDA study. J Infect Dis, 2001; 183: 1290-1294. DOI: 10.1086/319678.

24. Greenbaum AH, Wilson LE, Keruly JC, Moore RD, Gebo KA. Effect of age and HAART regimen on clinical response in an urban cohort of HIV-infected individuals. AIDS, 2008; 22: 2331-2339.

DOI: 10.1097/QAD.0b013e32831883f9.

25. Mateen FJ, Nath A. Central nervous systemimmune reconstitution inflammatory syndrome in resource-limited settings: current burden and future needs. AIDS, 2012; 26: 1851-1855. DOI: 10.1097/QAD.0b013e3283574e1a.

26. Dutertre M, Cuzin L, Demonchy E, Puglièse $P$, Joly V, Valantin MA, Cotte L, Huleux T, Delobel P, Martin-Blondel G; Dat'AIDS Study Group. Initiation of antiretroviral therapy containing integrase inhibitors increases the risk of IRIS requiring hospitalization. J Acquir Immune Defic Syndr, 2017; 76: e23-26. DOI: 10.1097/QAI.0000000000001397.

27. Psichogiou M, Basoulis D, Tsikala-Vafea M, Vlachos S, Kapelios CJ, Daikos GL. Integrase strand transfer inhibitors and the emergence of Immune Reconstitution Inflammatory Syndrome (IRIS). Curr HIV Res, 2017; 15: 405-410.

DOI: 10.2174/1570162X15666171122155708.

28. Wijting IEA, Wit FWNM, Rokx C, Leyten EMS, Lowe SH, Brinkman K, Bierman WFW, van Kasteren MEE, Postma AM, Bloemen VCM, Bouchtoubi G, Hoepelman AIM, van der Ende ME, Reiss P, Rijnders BJA; ATHENA national observational HIV cohort. Immune reconstitution inflammatory syndrome in HIV infected late presenters starting integrase inhibitor containing antiretroviral therapy. EClinicalmedicine, 2019; 17: 100210. DOI: 10.1016/j.eclinm.2019.11.003.

29. Tanaka $\mathrm{H}$, Ishii $\mathrm{T}$. Investigation of prescription pattern of antiretroviral using the national database of health insurance claims specific health checkups of Japan open data. J AIDS Res, 2019; 21: 173-180.

30. Poluzzi E, Raschi E, Moretti U, De Ponti F. Drug-induced torsades de pointes: data mining of the public version of the FDA Adverse Event Reporting System (AERS). Pharmacoepidemiol Drug Saf, 2009; 18: 512518. DOI: $10.1002 /$ pds.1746. 